\title{
CONVEXITY OF VECTOR-VALUED FUNCTIONS
}

\author{
IH-CHING HSU AND ROBERT G. KULLER
}

\begin{abstract}
Let $(B, \ll)$ be a Banach lattice, and $(a, b)$ be an open interval on the real line. A function $F:(a, b) \rightarrow \mathbb{B}$ is defined to be weakly convex if there exists a nonnegative nondecreasing continuous function $G:(a, b) \rightarrow B$ such that $p[F(S)]+t p[G(s)] \leq p[F(s+t)]$, whenever $s$ and $s+t$ are in $(a, b)$ for each positive linear functional $p$ on $B$. A representation theorem is proved as follows: If $F$ is weakly convex on $(a, b)$ and is bounded on an interval contained in $(a, b)$, then (B) $\int_{a+\epsilon}^{x} G(s) d m=F(x)-F(a+\epsilon)$, where $(B) \int_{a+\epsilon}^{x} G(s) d m$ is the Bochner integral of $G$ on $[a+\epsilon, x]$ with $0<\epsilon$ and $a+\epsilon<x<b$.
\end{abstract}

1. Introduction. Using a few facts in [1, pp. 91, 94, 95], an equivalent definition of convexity for continuous real-valued functions $f$ can be formulated as follows: $f$ is convex on an open interval $(a, b)$ if there exists a nondecreasing function $g$ on $(a, b)$ such that $f(s)+\operatorname{tg}(s) \leq f(s+t)$, whenever $s$ and $s+t$ are in $(a, b)$. Based on this equivalent definition, in [2], the first author gave a definition of weak convexity for functions $H:(a, b) \rightarrow$ $\mathfrak{Q}$, where $\mathcal{G}$ is a real commutative algebra closed in the strong topology of $\mathcal{H}(V, V)$, the space of all bounded hermitian operators on a Hilbert space $V$. The results proved in [2] rely on the fact that $\mathscr{Q}$ is a Dedekind complete lattice. Since $\mathbb{A}$ is also a Banach lattice in the sense given in [3, p. 366], a generalization of [2] to Banach lattices can be given; such is the purpose of this paper.

2. The weak convexity. A real Banach space $B$, with norm $\|\cdot\|$, is called a Banach lattice if $B$ is a vector lattice under a partial ordering « such that $|\beta| \ll|\alpha|$ implies $\|\beta\| \leq\|\alpha\|$ for each $\alpha$ and $\beta$ in $B$.

Definition 1. Let $(a, b)$ be an open interval of the real line, and $\theta$ be the zero vector in a Banach lattice $\mathcal{B}$. A function $F:(a, b) \rightarrow \mathcal{B}$ is weakly convex on $(a, b)$ if there exists a nondecreasing (w.r.t. $\gg$ ) and continuous

Presented to the Society, April 17, 1973; received by the editors February 7, 1973 and, in revised form, October 8, 1973.

AMS (MOS) subject classifications (1970). Primary 26A51; Secondary 46A40.

Key words and phrases. Weak convexity, Banach lattice, positive linear functional, Bochner integral, order-bounded, metric-bounded, Riesz decomposition theorem, weakly Lebesgue integrable, strongly Lebesgue measurable, Hahn-Banach theorem, strong convexity. 
(w.r.t. $\|\cdot\|$ ) function $G:(a, b) \rightarrow \mathcal{B}$ such that $G(s) \gg \theta, s \in(a, b)$, and $p[F(s)]+t p[G(s)] \leq p[F(s+t)]$, whenever $s$ and $s+t$ are in $(a, b)$ for each positive linear functional $p$ on $\mathcal{B}$.

Theorem. Suppose that $F:(a, b) \rightarrow B$ is weakly convex on $(a, b)$. If $F$ is bounded on an interval $J \subset(a, b)$ in the sense that there exists a $K$ in $B$ such that $|F(t)|=F^{+}(t)+F^{-}(t) \ll K$ for each $t$ in $J$, then

$$
\text { (B) } \int_{a+\epsilon}^{x} G(s) d m=F(x)-F(a+\epsilon) \text {, }
$$

where $\epsilon>0$ and $a+\epsilon \leq x<b$, and $(B) \int_{a+\epsilon}^{x} G(s), d m$ is the Bochner integral of $G$ on $[a+\epsilon, x]$ with respect to the Lebesgue measure $m$.

Proof. It is known [3, p. 368] that order-boundedness and metricboundedness are equivalent for linear functionals on a Banach lattice. Thus the space $\mathfrak{B}^{b}$ of all order-bounded linear functionals on the vector lattice $\mathscr{B}$ is the same as the space $\mathfrak{B}^{*}$ of all norm continuous linear functionals on the Banach space $\mathbb{B}$.

Let $q$ be an element in $\mathfrak{B}^{b}=\mathfrak{B}^{*}$. Then by the well-known Riesz decomposition theorem [5, p. 68], $q=q^{+}-q^{-}$, where $q^{+}$and $q^{-}$are positive linear functionals on $\mathcal{B}$. The weak convexity of $F$ now implies that

$$
q^{+}[F(s)]+t q^{+}[G(s)] \leq q^{+}[F(s+t)], \quad s, s+t \in(a, b) .
$$

The real-valued function $q^{+} \circ F$ is bounded on $J$, since

$$
\left|q^{+}[F(s)]\right|=\left|q^{+}\left[F^{+}(s)\right]-q^{+}\left[F^{-}(s)\right]\right| \leq 2 q^{+}(K)
$$

for each $s$ in $J$. To simplify notations, let $q^{+} \circ F=f, q^{+} \circ G=g$, and rewrite (1) as follows:

$$
\begin{aligned}
& f(s)+\operatorname{tg}(s) \leq f(s+t) \quad \text { whenever } s \text { and } s+t \in(a, b) ; \text { or } \\
& f(s)-\operatorname{tg}(s) \leq f(s-t) \quad \text { whenever } s \text { and } s-t \in(a, b) .
\end{aligned}
$$

It follows from (2) and (3) that

$$
2 f(s) \leq f(s+t)+f(s-t) \text { whenever } s+t \text { and } s-t \in(a, b) \text {. }
$$

This is equivalent to $f(1 / 2 s+1 / 2 t) \leq 1 / 2 f(s)+1 / 2 f(t)$ whenever $s$ and $t$ are in $(a, b)$. Since $f$ is convex and bounded on $J, f$ is continuous on $(a, b)[1$, p. 91]. From (2) and (3) it follows that for positive small $\delta, f(s-\delta)+$ $\delta g(s-\delta) \leq f(s)$ and $f(s)-\delta g(s) \leq f(s-\delta)$. Therefore, $g$ is nondecreasing on $(a, b)$.

For positive small $t$, rewrite (2) as $g(s) \leq(f(s+t)-f(s)) / t$ and integrate both sides of this inequality over $[a+\epsilon, x], \epsilon>0, a+\epsilon \leq x<b$, to obtain 


$$
\int_{a+\epsilon}^{x} g(s) d s \leq \frac{\int_{a+\epsilon}^{x} f(s+t) d s-\int_{a+\epsilon}^{x} f(s) d s}{t} .
$$

Clearly, the reverse inequality holds for negative small $t$ as follows:

$$
\int_{a+\epsilon}^{x} g(s) d s \geq \frac{\int_{a+\epsilon}^{x} f(s+t) d s-\int_{a+\epsilon}^{x} f(s) d s}{t} .
$$

Passing to the limit as $t \rightarrow 0,(5)$ and (6) yield

$$
\int_{a+\epsilon}^{x} g(s) d s=f(x)-f(a+\epsilon)
$$

i.e.

$$
\int_{a+\epsilon}^{x} q^{+}[G(s)] d s=q^{+}[F(x)]-q^{+}[F(a+\epsilon)]
$$

Similarly,

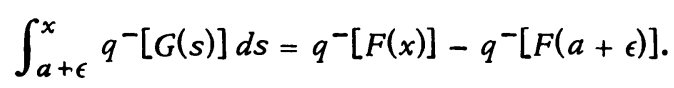

Thus for each bounded linear functional $q \in \mathfrak{B}^{*}$,

$$
\begin{aligned}
\int_{a+\epsilon}^{x} q[G(s)] d s & =\int_{a+\epsilon}^{x}\left(q^{+}-q^{-}\right)[G(s)] d s \\
= & \left(q^{+}-q^{-}\right)[F(x)]-\left(q^{+}-q^{-}\right)[F(a+\epsilon)] \\
= & q[F(x)]-q[F(a+\epsilon)] .
\end{aligned}
$$

From (7) it is clear that $G(s)$ is weakly Lebesgue integrable on $[a+$ $\epsilon, x]$. Moreover, $G(s)$ is strongly Lebesgue measurable on $[a+\epsilon, x]$, since $G[a+\epsilon, x]$, the range of $G$, is separable in $B$ because of the continuity of $G[4$, p. 131]. The Bochner integral of $G$ exists if the strongly Lebesgue measurable function $G(s)$ has the property that $\|G(s)\|$ is Lebesgue integrable [4, p. 133]. By assumption, with respect to $\ll, G$ is nondecreasing on $[a+\epsilon, x]$ with $\theta \ll G(a+\epsilon) \ll G(x)$ in the Banach lattice $B$. Thus $\|G(s)\|$ is nondecreasing on $[a+\epsilon, x]$ and, therefore, Lebesgue integrable. Let (B) $\int_{a+\epsilon}^{x} G(s) d m$ denote the Bochner integral of $G$ over $[a+\epsilon, x]$; then

$$
\text { (B) } \int_{a+\epsilon}^{x} G(s) d m=F(x)-F(a+\epsilon)
$$

by (7) and the Hahn-Banach theorem. This completes the proof of the theorem.

3. The strong convexity. The notion of strong convexity for functions $F:(a, b) \rightarrow \mathcal{B}$ can be formulated as follows:

Definition 2. $F:(a, b) \rightarrow \Re$ is strongly convex on $(a, b)$ if there exists 
a nondecreasing continuous function $G:(a, b) \rightarrow \mathcal{B}$ such that $G(t) \gg \theta$ for each $t$ in $(a, b)$ and $F(s)+t G(s) \ll F(s+t)$, whenever $s$ and $s+t$ are in $(a, b)$.

Clearly, the strong convexity implies the weak convexity. The validity of the converse statement is an open question. One natural way to proceed is to check whether or not $F(x)=F(a+\epsilon)+(B) \int_{a+\epsilon}^{x} G(s) d m$ satisfies $F(s)+t G(s) \ll F(s+t)$. The difficulty is that we do not know that the continuity and monotonicity of $G$ imply $(B) \int_{x}^{x+y} G(s) d m \gg y G(x)$.

Added in proof. The problem specifically mentioned here has been solved positively in [6].

\section{REFERENCES}

1. G. H. Hardy, J. E. Littlewood and G. Pólya, Inequalities, 2nd ed., Cambridge Univ. Press, London and New York, 1952. MR 13, 727.

2. I. Hsu, Weak convexity of operator-valued functions (unpublished).

3. G. Birkhoff, Lattice theory, 3rd ed., Amer. Math. Soc. Colloq. Publ., vol. 25, Amer. Math. Soc., Providence, R. I., 1967. MR 37 \#2638.

4. K. Yosida, Functional analysis, Die Grundlehren der math. Wissenschaften, Band 123, Springer-Verlag, Berlin and New York, 1965. MR 31 \#5054.

5. G. Jameson, Ordered linear spaces, Springer-Verlag, Berlin and New York, 1970.

6. I. Hsu, A functional inequality and its relation to convexity of vector-valued functions (submitted).

DEPARTMENT OF MATHEMATICS, ST. OLAF COLLEGE, NORTHFIELD, MINNESOTA 55057

DEP ARTMENT OF MATHEMATICAL SCIENCES, NORTHERN ILLINOIS UNIVERSITY, DEKALB, ILLINOIS 60115 